\title{
Article \\ A Steganalysis Classification Algorithm Based on Distinctive Texture Features
}

\author{
Baraa Tareq Hammad ${ }^{1}$, Ismail Taha Ahmed ${ }^{1, *}$ and Norziana Jamil ${ }^{2, *}$ \\ 1 College of Computer Sciences and Information Technology, University of Anbar, Ramadi 55431, Iraq; \\ baraa.tareq@uoanbar.edu.iq \\ 2 College of Computing and Informatics, Universiti Tenaga Nasional, Kajang 43000, Selangor, Malaysia \\ * Correspondence: ismail.taha@uoanbar.edu.iq (I.T.A.); Norziana@uniten.edu.my (N.J.)
}

Citation: Hammad, B.T.; Ahmed, I.T.; Jamil, N. A Steganalysis

Classification Algorithm Based on

Distinctive Texture Features.

Symmetry 2022, 14, 236. https://

doi.org/10.3390/sym14020236

Academic Editor: László T. Kóczy

Received: 13 November 2021

Accepted: 15 December 2021

Published: 25 January 2022

Publisher's Note: MDPI stays neutral with regard to jurisdictional claims in published maps and institutional affiliations.

Copyright: (C) 2022 by the authors. Licensee MDPI, Basel, Switzerland. This article is an open access article distributed under the terms and conditions of the Creative Commons Attribution (CC BY) license (https:// creativecommons.org/licenses/by/ $4.0 /)$.

\begin{abstract}
Steganography is the technique for secretly hiding messages in media such as text, audio, image, and video without being discovered. Image is one of the most essential media for concealing data, making it hard to identify hidden data not visible to the human eye. In general, the cover image and the encrypted image are symmetrical in terms of dimension size, resolution, and qualities. This makes the difference difficult to perceive with the human eye. As a result, distinguishing between the two symmetric images required the development of methods. Steganalysis is a technique for identifying hidden messages embedded in digital material without having to know the embedding algorithm or the "non-stego" image. Due to their enormous feature vector dimension, which requires more time to calculate, the performance of most existing image steganalysis classification (ISC) techniques is still restricted. Therefore, in this research, we present a steganalysis classification method based on one of the texture features chosen, such as segmentation-based fractal texture analysis (SFTA), local binary pattern (LBP), and gray-level co-occurrence matrix (GLCM). The classifiers employed include Gaussian discriminant analysis (GDA) and naïve Bayes (NB). We used a public database in our proposed method and applied it to IStego100K datasets to be able to assess its performance. The experimental results reveal that in all classifiers, the SFTA feature surpassed all of the texture features, making it a great texture feature for image steganalysis classification. In terms of feature dimension and classification accuracy (CA), a comparison was made between the suggested SFTA-based GDA approach and various current ISC methods. The outcomes of the comparison are obvious show that the proposed method surpasses current methods.
\end{abstract}

Keywords: image steganalysis classification (ISC); LBP; SFTA; Gaussian discriminant analysis (GDA) classifier; naïve Bayes (NB) classifier

\section{Introduction}

Due to the rapid growth of social networking sites, we may see or receive a large number of photographs, but we have no way of knowing whether these images are original or encrypted. Steganography is a method of hiding private information in media such as text, audio, image, and video without leaving any trace that they are encrypted, as shown in Figure 1. The figure shows that the cover image and the encrypted image, in general, are symmetrical in terms of size dimension, resolution, and qualities. Therefore, we urgently require methods to distinguish photos containing an encrypted object. The goal of blind steganalysis is to detect steganographic data without knowing the embedding algorithm or the cover image.

Figure 2 depicts a general taxonomy of steganalysis techniques, which is separated under multimedia data types and domains. Steganalysis approaches are classified into two kinds, signature and statistical steganalysis, according to steganalysis detection methods in the literature review. Statistical steganalysis is the process of seeking to find such statistical traces. When compared with signature steganalysis, statistical steganalysis 
is a more powerful tool since mathematical procedures are more sensitive than visual perception $[1,2]$.

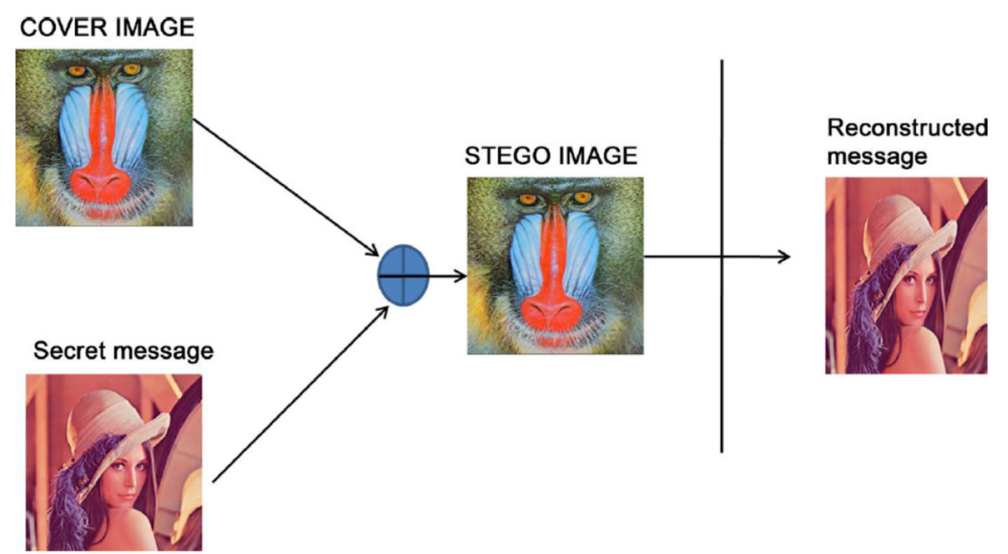

Figure 1. General architecture of steganography [1].

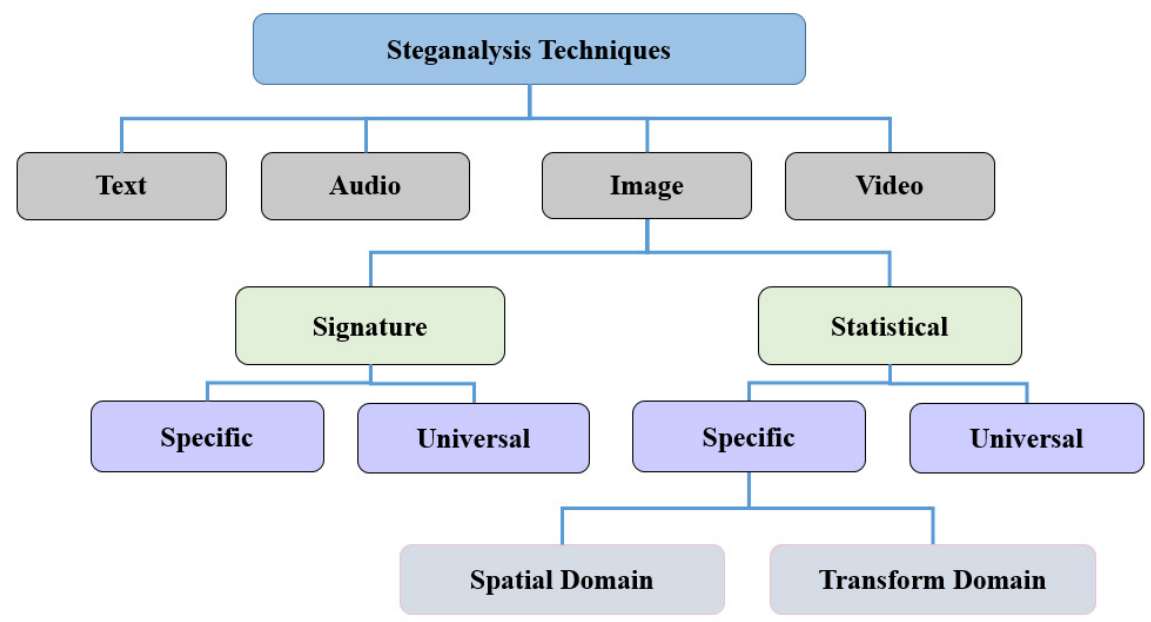

Figure 2. Steganalysis techniques classification.

The majority of steganalysis approaches rely on image statistical calculations such as first and second-order statistics. Statistical and signature steganalysis can be divided into two categories: specific and universal. Specific steganalysis is created for a particular steganographic embedding algorithm, such as LSB embedding, LSB matching, spread spectrum, BPCS, JPEG compression, and other transform domains [3], whereas universal steganalysis is a general class steganalysis technique that can be used with any steganographic embedding algorithm, including unknown algorithms [4].

Most of the previous image steganalysis classification (ISC) methods suffer from a poor classification rate. In order to improve the accuracy and reduce the high dimensionality of extracted features, we proposed an image steganalysis classification (ISC) method based on three steps: First was the pre-processing stage, after which texture features were extracted by using SFTA, LBP, and GLCM. Finally, the classifiers Gaussian discriminant analysis (GDA) and naïve Bayes (NB) were chosen.

The rest of the article is arranged as follows: The relevant works are presented in Section 2. Some texture analysis techniques are discussed in Section 3. The presented ISC methods are detailed in Section 4. Section 5 discusses experimental results and analysis. Finally, in Section 6, conclusions are drawn.

\section{Related Works}

Several studies on the subject of steganalysis have been conducted in recent years and are still ongoing. The majority of image steganalysis techniques described in the 
literature have been primarily concerned with either the spatial or transform domains. For information hiding, spatial domain approaches rely entirely on the pixels of the image. As a result, spatial domain approaches are easy and quick to implement. The frequency content is used in transform domain techniques, which are based on the orthogonal frequency and phase transformations of the image. These techniques are complicated. In the spatial domain, multiple image steganalysis classification techniques (ISCTs) are introduced.

Ashu et al. [5] proposed an image steganalysis classification (ISC) method that relied on GLCM, as well as J48 and SMO, and the naïve Bayes was used as the classifier. Gui et al. [6] proposed an ISC method based on LBP. In summary, smooth pixels were used to extract multi-scaled rotation invariant LBPs as distinguishing features. After that, linear SVM was used to train and classify the features. Wang and Gong [7] proposed an ISC method that relied on the cloud gray-level co-occurrence matrix (CGCM) for GIF images. In this method, 27-Dimensional statistical features of CGCM were color-correlation sensitive. In [8], Arooj Nissar et al. presented an ISC method that relied on the spatial gray-dependency technique. The texture info from the non-stego and stego images was utilized to train a neural network classifier, which was then applied to test images. Tao Zhang et al. [9] presented an ISC method that relied on statistical analyses of differential image histograms. Song et al. [10] presented an ISC method relying on the Shannon entropy of 2D Gabor wavelets. An ensemble classifier was utilized for classification. Karimi et al. [11] presented an ISC method that relied on discrete cosine transform (DCT) coefficients. An ensemble classifier was utilized for classification. Jyothy et al. [12] presented an ISC method that relied on different textural features Such as GLCM, discrete wavelet transform (DWT), and contourlet transform (CT). For classification, the AdaBoost classifier was utilized. Liu et al. [13] presented an ISC method that relied on generalized Gaussian distribution (GGD) in the wavelet domain. Lin et al. [14] presented an ISC method relying on LTP and pi-LBP features combined. After evaluating the literature on the ISC technique, we concluded that more effort should be invested in this area. This is primarily to improve accuracy and reduce the large dimensionality of extracted features, hence increasing the time complexity. The feature discrimination can be improved by combining features. For classification, an ensemble classifier was used.

\section{Texture Analysis}

Texture in an image provides information about the spatial arrangement of colors or intensities. The spatial distribution of intensity levels in a neighborhood defines texture. Texture analysis is important in a number of situations. One of the most important is steganalysis [15]. Texture analysis is used to uncover information that the human eye cannot perceive since the information buried in the images is very difficult to identify or discriminate with the naked eye. In the case of embedding the secret data in an image, the texture and features of the image are drastically altered. As a result, texture analysis may be able to easily reveal these buried features. In this section, some of the texture features include SFTA, LBP, and GLCM are employed to extract texture features. In what follows, each texture descriptor is explored in detail.

\section{1. $L B P$}

The primary contribution of the original LBP operator [16] is the marking of image pixels with decimal values, which are referred to as LBPs and represent the local structure around pixels [6]. In particular, each central pixel is initially compared in value with its eight neighbors in a $3 \times 3$ square context. The relevant place is indicated as 1 if the adjacent pixel is not less than the center one, and 0 otherwise. A binary number can be derived as the LBP value for the central pixel by appending every one of these binary values in a clockwise direction from its top-left neighbor. Lastly, the resulting binary number's decimal 
value is utilized to mark the specified pixel. The generalized LBP $[6,14]$ can be stated in decimal notation as follows:

$$
\operatorname{LBP}_{P, R}=\sum_{P=0}^{P} s\left(x_{P}-c\right) 2^{P}
$$

When $x$ is less than zero, the function $\mathrm{s}(x)$ equals 1 ; otherwise, it equals 0. In the example shown in Figure 3, the central pixel value is 15, and the pixels in the 8 nearby pixels that are not less than 15 represent 1 in the associated LBP, while the others indicate 0 . As a result, the LBP received is 11000111, with a decimal value of 199.

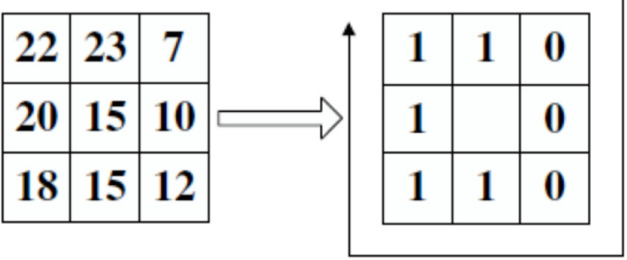

Binary: 11000111

Decimal: 199

Figure 3. The local binary pattern (LBP) operator example [6].

The stego signal causes a disruption in the correlation of adjacent pixels in the cover image, which can be used for steganalysis. The LBP is a useful image texture feature for describing the relationship between adjacent pixels, which is the main argument for employing it.

\subsection{SFTA}

One of the most common texture descriptors is segmentation-based fractal texture analysis (SFTA) [17]. The texture of an image is its most noticeable feature. The SFTA method has two major phases: (i) using the two-threshold binary decomposition (TTBD) method to decompose the input grayscale image as a set of binary images; (ii) for each binary image formed by subtracting the fractal dimension from the borders of its regions, SFTA feature vectors are computed. For more details, see [17]. The SFTA features are extracted using the given mathematical formula (Equation (2)).

$$
\varnothing_{\text {sfta }}(U)=\left\{\begin{array}{c}
1 \text { if } \exists\left(i^{\prime}, j^{\prime}\right) \in N_{8}[(i, j)]: \\
\varnothing_{e}\left(i^{\prime}, j^{\prime}\right)=0^{\prime} \\
\varnothing_{e}(i, j)=1 \\
\text { 0 Otherwise }
\end{array}\right.
$$

where $N_{8}[(i, j)]$ represents the number of connected pixels, which is set at eight. A Binary image is $\varnothing_{e}(i, j)$. In order to define the dimension of the features vector, the number of thresholds is determined. If the number of thresholds is set to three, seven binary images are generated, and then, 21 features are produced for each image.

Due to their durability and low computing cost, SFTA features are employed for texture feature extraction among texture image analysis methods. As a result, applying SFTA features extraction to image steganalysis classification is intriguing.

\subsection{GLCM}

Gray-level co-occurrence matrix (GLCM) is a powerful second-order statistical approach for analyzing texture. For each image, GLCM is the matrix containing information about the relationship between values of an adjacent pixel $[18,19]$. Texture descriptors, or GLCM descriptors, are common texture features used to extract texture information. The GLCM [20] features are dependent on statistical moments and are obtained from a cooccurrence matrix. Figure 4 shows an example of GLCM. In total, 14 textural characteristics were computed from using GLCM. 


Image matrix
\begin{tabular}{|l|l|l|l|}
\hline 0 & 0 & 1 & 1 \\
\hline 0 & 0 & 1 & 1 \\
\hline 0 & 2 & 2 & 2 \\
\hline 2 & 2 & 3 & 3 \\
\hline
\end{tabular}$\Rightarrow$\begin{tabular}{|l|l|l|l|l|}
\hline$i j$ & 0 & 1 & 2 & 3 \\
\hline 0 & $\#(0,0)$ & $\#(0,1)$ & $\#(0,2)$ & $\#(0,3)$ \\
\hline 1 & $\#(1,0)$ & $\#(1,1)$ & $\#(1,2)$ & $\#(1,3)$ \\
\hline 2 & $\#(2,0)$ & $\#(2,1)$ & $\#(2,2)$ & $\#(2,3)$ \\
\hline 3 & $\#(3,0)$ & $\#(3,1)$ & $\#(3,2)$ & $\#(3,3)$ \\
\hline
\end{tabular}

Figure 4. An example of GLCM.

GLCM depicts the number of times pixel $I$ at location $(x, y)$ occurs in accordance with pixel $j$ at position $(x+x, y+y)$ for a given image $I(x, y)$ of size $M \times N$ with Gt as total different gray levels. The frequency of occurrence is denoted by $A(i, j, d)$ and is mathematically formulated by

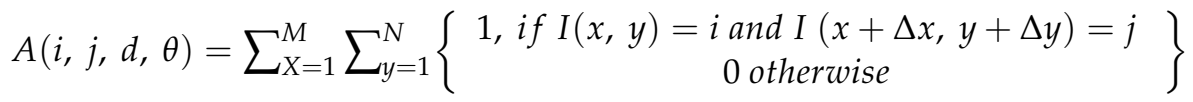

where ' $d$ ' specifies the pixel's $x, y$ offset distance from its neighbor and signifies the direction. More details can be found in $[20,21]$. Each stego image is subjected to the GLCM feature extraction method, which extracts distinguishing characteristics that are utilized to improve feature extraction efficiency.

\section{The Proposed Methods}

This section includes a flowchart of the proposed method, as illustrated by Figure 5 . Furthermore, the Algorithm 1 was created. The following are the descriptions of the steps in the algorithm:

Algorithm 1: Proposed Steganalysis Classification_based GDA and Naïve Bayes Classifier. INPUT: Image Dataset.

OUTPUT: Non-stego/Stego Image.

\section{Begin}

For

1: Read each image by "Imread ( )" function;

2: Transforming the RGB image to the gray image using "rgb2gray ( $)$ " function;

3: For each transformed image, extract the SFTA features \{Sftaf1, Sftaf2, Sftaf3, Sftaf4, ...

Sftaf21\} to obtain a 21-dimension feature vector;

4: For each transformed image, extract the LBP features $\{$ Lbpf1, Lbpf2, Lbpf3, Lbpf4 ...

Lbpf59\} to obtain a 59-dimension feature vector;

5: Extract the GLCM features vector:

A. Create the co-occurrence matrix for each transformed image, using "graycomatrix ( )" function;

B. Extract the GLCM features \{Glcmf1, Glcmf2, Glcmf3, Glcmf4 ... Glcmf14\} to obtain a 14-dimension feature vector.

6: Training:

A. Train the GDA classifier with these feature vectors;

B. Train the naïve Bayes classifier with these feature vectors.

7: Testing:

A. Test the trained GDA model to determine if the image is non-stego or stego;

B. Test the trained naïve Bayes model to determine if the image is non-stego or stego.

End for

End 


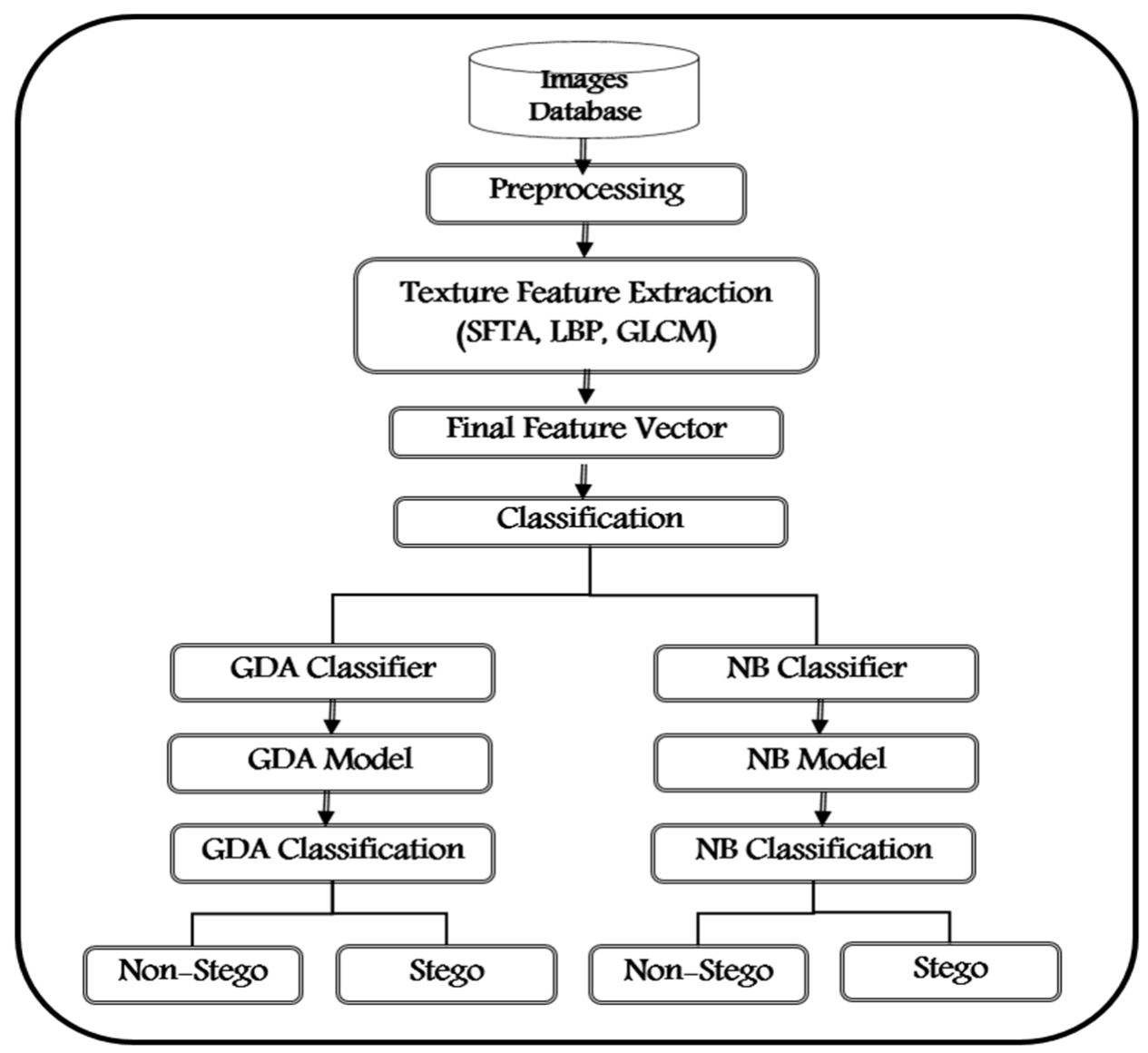

Figure 5. The proposed method's flowchart.

\subsection{Pre-Processing}

The input, a colored image $\mathrm{I}(x, y)$, was transformed into a grayscale image using the "rgb2gray $(\mathrm{I}(x, y))$ " function during the pre-processing stage. The objective was to decrease the computational burden. The secret information is not destroyed as a result of this processing.

\subsection{Texture Feature Extraction}

The method of obtaining the relevant information or features from the original image is known as feature extraction. The texture features SFTA, LBP, and GLCM were extracted for each cover and stego image, as illustrated in Figure 6. As detailed in the following, the final feature vector dimension was 94. The final SFTA feature vector extracted is as follows: (Sftaf1, Sftaf2, Sftaf3, Sftaf4, ... Sftaf21). The SFTA feature vector had a dimension of 1 $\times 21$. The final LBP feature vector extracted is as follows (Lbpf1, Lbpf2, Lbpf3, Lbpf4 ... Lbpf59). The LBP feature vector had a dimension of $1 \times 59$. The final GLCM feature vector extracted is as follows (Glcmf1, Glcmf2, Glcmf3, Glcmf4 ... Glcmf14). The GLCM feature vector had a dimension of $1 \times 14$. The final combined feature vector of SFTA, LBP, and GLCM had a dimension of $1 \times 94$. 


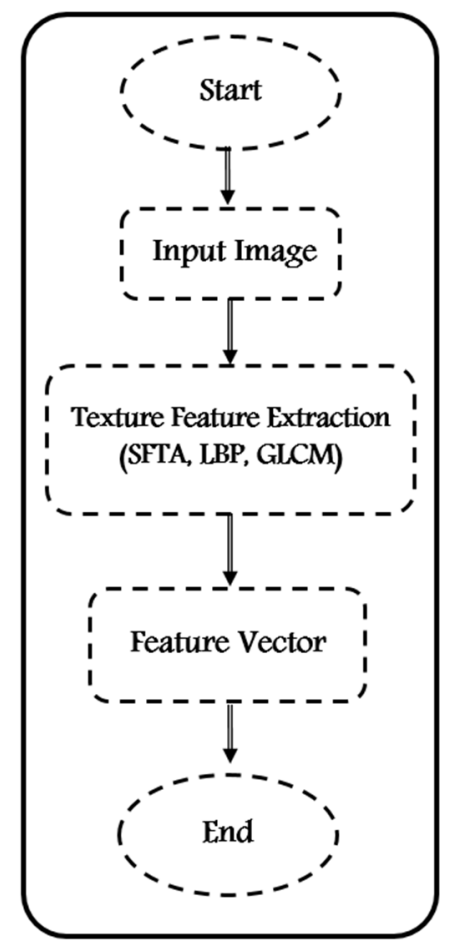

Figure 6. Feature extraction flowchart.

\subsection{Classification}

Classification is a fundamental stage that involves extracting image features from an original image (non-stego image) and its stego counterpart. The aim would be to discern the difference between non-stego or stego-image. As a result, in this paper, the GDA and naive Bayes (NB) classifiers were employed to identify whether the image is non-stego or stego, as shown in Figure 5. The classifiers were fed the feature vector derived from the SFTA, LBP, and GLCM features. In the training phase, the two classifiers were trained with various texture-related feature sets. The trained classifiers were used to identify a stego image from a non-stego image during the testing phase.

In order to perform the classification problem, the Gaussian discriminant analysis (GDA) [22,23] is the most well generative model. The naïve Bayes classifier is built on Bayes' theorem. This classifier is regarded as a straightforward probabilistic one. The basic function of this classifier is to produce a set of probabilities for each dataset by calculating the frequency and combinations of values [24].

\section{Experimental Results and Analysis}

In order to demonstrate the efficacy of the proposed method, this section contains the following: (i) MATLAB 2020 (b) was used for the experimental and assessment of the proposed method; (ii) images from publicly available websites were used to generate a database for training and testing; (iii) the performance analysis and results were displayed; (iv) using various classifiers, the proposed methods were compared with the state of the art.

\subsection{Dataset}

IStego100K (large-scale image steganalysis dataset) [25] is a public dataset that contains 208,104 images. The training set consists of 200,000 images $(100,000$ cover-stego image pairings), while the testing set consists of 8104 images. The cover/stego images have a resolution of $1024 * 1024$. The databases for cover and stego image samples are shown in Figure 7. 

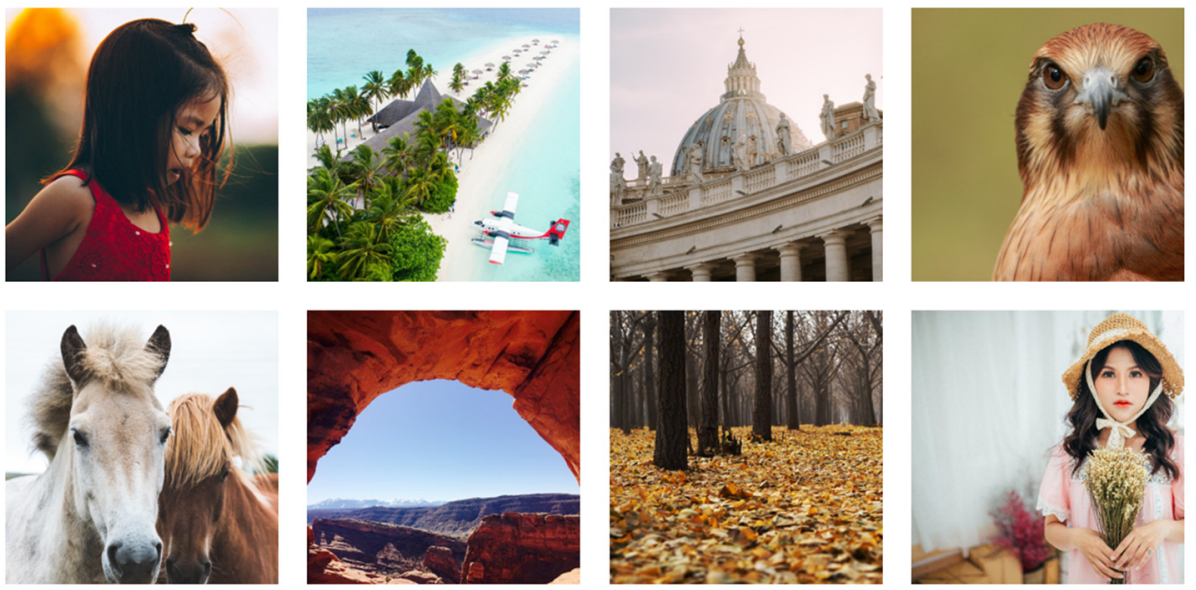

Figure 7. The first row is the original image (non-stego); the second row is stego image.

\subsection{Performance Results}

\subsubsection{Performance Evaluation}

Equation (4) can be used to calculate the classification accuracy, which is the percentage of non-stego and stego images that are correctly classified to the total number of non-stego and stego images.

$$
\text { Classification Accuracy }=\frac{(T p+T n)}{(T p+T n+F p+F n)} \times 100 \%
$$

True positive indicates the number of correctly categorized stego images $(T p)$. The number of false negatives $(F n)$ indicates how many stego images were erroneously classified. False positives are the number of wrongly classified non-stego images $(F p)$. True negative indicates the number of correctly categorized non-stego images (Tn).

\subsubsection{Evaluation Results}

As indicated in Table 1, the performance analysis for each classifier is listed in order to reveal whether a test image is stego or non-stego. In order to select the best classifier, the accuracy of GDA and NB classifiers was measured.

Table 1. The accuracy results of different classifiers and features.

\begin{tabular}{ccccc}
\hline \multirow{2}{*}{ Classifier } & \multicolumn{5}{c}{ Texture Features } \\
\cline { 2 - 5 } & LBP & GLCM & SFTA & Combined Features \\
\cline { 2 - 5 } & \multicolumn{5}{c}{ Detection Accuracy (\%) } \\
\hline GDA & 75 & 79 & 90 & 85 \\
\hline Naive Bayes & 71 & 73 & 75 & 75 \\
\hline
\end{tabular}

Despite the fact that all classifiers used the same feature vector, they yielded different outputs. This is due to the fact that each classifier has its own range of attributes. The performance of each classifier is detailed here. As shown in Table 1, the accuracy of the GDA classifier for LBP, GLCM, SFTA, and combined features was 75, 79, 90, and 85, respectively. As a result, it is reasonable to claim that the GDA classifier outperformed the naïve Bayes classifier in terms of accuracy rate, with an accuracy rate of $90 \%$. As shown in Table 1 , the accuracy of the NB classifier for LBP, GLCM, SFTA, and combined features was 71, 73,75 , and 75, respectively. As a consequence, behind the GDA classifier, the naïve Bayes classifier came in second. Figure 8 shows a graphical representation of the accuracy rate of two classifiers, GDA and naïve Bayes, which were used to categorize test images using 
various texture features. As shown in Figure 8, we conclude that when employing the GDA classifier, SFTA produced significant results in terms of the accuracy rate.

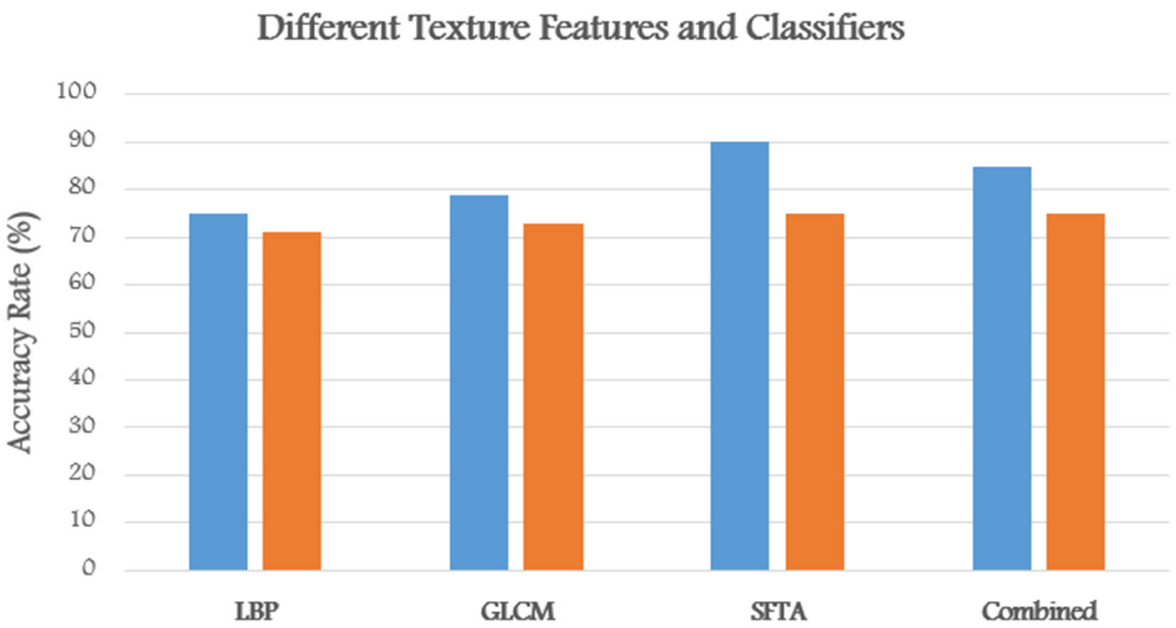

GDA Classifier $\square$ NB Classifier

Figure 8. Graphical representation of the accuracy rate of different texture features and classifiers.

In both classifiers, when compared with LBP and GLCM features, the SFTA feature emerged on top, as seen in Table 1. While the performance of GLCM came after the SFTA feature. The LBP feature had a lower performance than the other texture features. Furthermore, experimental results demonstrate that combining features (LBP, GLCM, SFTA) did not improve performance over using separate features (LBP, GLCM, and SFTA). When compared with a combination of feature vectors, an individual feature vector using SFTA could attain an accuracy of $90 \%$.

Lastly, we conclude that the proposed method, which relied on the SFTA texture feature and the GDA classifier, outperformed all other texture features and classifiers, making it a great texture feature for steganalysis classification.

\subsection{Comparison with Previous ISC Works}

In this section, we compare our method to other methods in order to assess its performance. The extraction of texture features is the main element of each of these image steganalysis classification algorithms. The proposed approach is compared with existing methods relying on feature-based steganalysis in Table 2 . The comparison shows that the proposed method outperforms current state-of-the-art methods in terms of classification rates.

Table 2. Comparison of performance with other ISC techniques.

\begin{tabular}{|c|c|c|c|c|}
\hline Techniques & Feature Vector Dimension & Texture Features & Classifier & Accuracy (\%) \\
\hline Jyothy et al. [12] & 416 & GLCM, DWT, and CT & Ada-Boost & 93 \\
\hline Qin et al. [26] & 22130 & GLCM & Ensemble Classifier & 83 \\
\hline Chhikara et al. [5] & 134 & $\begin{array}{c}\text { Global histogram and } \\
\text { GLCM }\end{array}$ & $\begin{array}{l}\text { Naïve } \\
\text { Bayes }\end{array}$ & 60 \\
\hline Lin et al. [14] & 1944 & LBP & Ensemble Classifiers & 94 \\
\hline Proposed & 21 & SFTA & GDA & 90 \\
\hline
\end{tabular}

As demonstrated in Figure 9, the proposed feature extraction strategy has a better accuracy rate and fewer feature vector dimensions (21) than the majority of existing approaches, making it computationally easier. The methods $[12,14]$ produced excellent results, 
with 416 and 1944 feature vector dimensions, respectively. However, the feature vector's high dimension necessitates a significant amount of computation. Ultimately, the experiment results in Table 2 show that our method uses fewer features and improves image steganalysis classification accuracy.

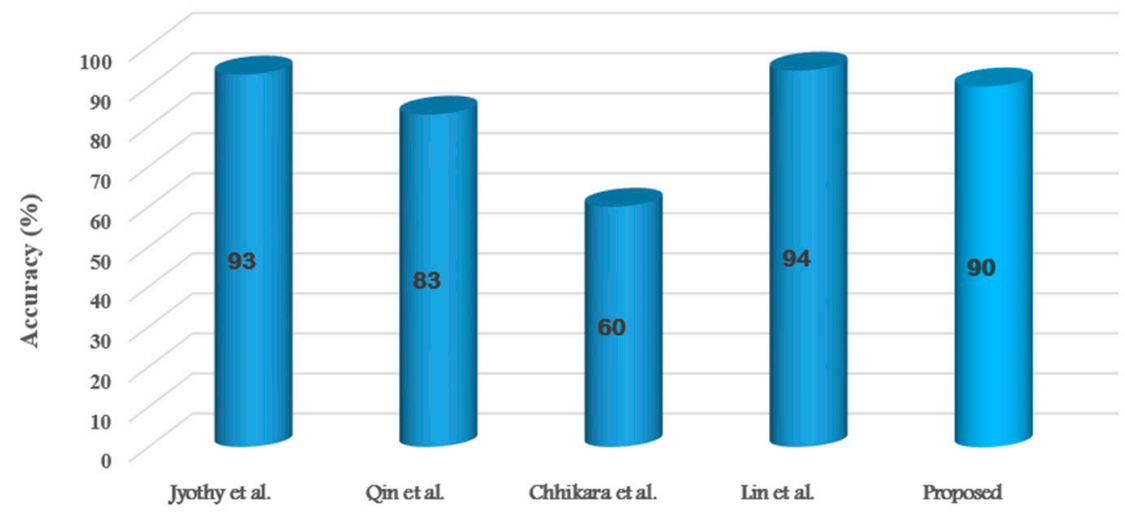

Figure 9. Performance Assessment based on several features and classifiers.

\section{Conclusions}

The main objective of this research was to find efficient and trustworthy texture feature extraction methods and classifiers for improved steganalysis performance and a high classification rate. First image preprocessing was carried out, followed by texture feature extraction techniques. SFTA, LBP, and GLCM were used to extract texture feature values, which were then utilized to train two distinct classifiers, GDA and naïve Bayes, to identify a stego image from a non-stego image.

The experimental results show that the accuracy of the GDA classifier for LBP, GLCM, and SFTA, as well as combined features, was 75, 79, 90, and 85, respectively. Therefore, it is reasonable to claim that the GDA outperformed the naïve Bayes classifier in terms of accuracy rate, with an accuracy rate of $90 \%$. The accuracy of the NB classifier for LBP, GLCM, and SFTA, as well as combined features, was 71, 73, 75, and 75, respectively. As a consequence, behind the GDA classifier, the naïve Bayes classifier came in second. It can be seen that when employing the GDA classifier, SFTA surpassed LBP and GLCM in terms of accuracy rate.

GDA had the best classification rate, with 90 percent, among the classifiers. The suggested method outperformed other existing texture-based analysis methods in terms of feature vector dimension and classification accuracy (CA), according to the results. Experiments results show that the presented method, which relied on the SFTA texture feature and the GDA classifier, surpassed LBP and GLCM features and NB classifier, making it a great texture feature for image steganalysis classification. The most current approaches have more features vector dimensions than the proposed feature extraction technique, making the method computationally simpler. In the future, we will look into approaches such as Gabor that can successfully capture changes in image texture. We also aim to use deep learning to implement the method.

Author Contributions: Conceptualization, I.T.A., B.T.H. and N.J.; writing—original draft preparation, I.T.A.; validation, I.T.A., B.T.H.; writing—review and editing, I.T.A., B.T.H., N.J. All authors have read and agreed to the published version of the manuscript.

Funding: This research is supported by the United BOLD Publication Fund 2021.

Institutional Review Board Statement: Not applicable.

Informed Consent Statement: Not applicable.

Data Availability Statement: https:/ / github.com/YangzlTHU/IStego100K (accessed on 10 September 2021). 
Acknowledgments: This research is supported by Uniten BOLD Publication Fund 2021.

Conflicts of Interest: The authors declare no conflict of interest.

\section{References}

1. Babu, J.; Rangu, S.; Manogna, P. A survey on different feature extraction and classification techniques used in image steganalysis. J. Inf. Secur. 2017, 8, 186. [CrossRef]

2. Chanu, Y.J.; Singh, K.M.; Tuithung, T. Image steganography and steganalysis: A survey. Int. J. Comput. Appl. 2012, 52, 1-11.

3. Mandal, P.C.; Poddar, B.P. An extensive review of current trends in steganalysis. Int. J. Adv. Res. Comput. Eng. Technol. 2012, 1, 215.

4. Nissar, A.; Mir, A.H. Classification of steganalysis techniques: A study. Digit. Signal Process. 2010, 20, 1758-1770. [CrossRef]

5. Chhikara, R.R.; Bansal, D. GLCM based features for steganalysis. In Proceedings of the 2014 5th International ConferenceConfluence The Next Generation Information Technology Summit (Confluence), Noida, India, 25-26 September 2014; pp. 385-390.

6. Gui, X.; Li, X.; Yang, B. Steganalysis of LSB matching based on local binary patterns. In Proceedings of the 2014 Tenth International Conference on Intelligent Information Hiding and Multimedia Signal Processing, Kitakyushu, Japan, 27-29 August 2014; pp. $475-480$.

7. Gong, R.; Wang, H. Steganalysis for GIF images based on colors-gradient co-occurrence matrix. Opt. Commun. 2012, 285, 4961-4965. [CrossRef]

8. Nissar, A.; Mir, A.H. Texture based steganalysis of grayscale images using neural network. Signal Process. Res. 2013, 2, 17-24.

9. Zhang, T.; Ping, X. A new approach to reliable detection of LSB steganography in natural images. Signal Process. 2003, 83, 2085-2093. [CrossRef]

10. Song, X.; Li, Z.; Chen, L.; Liu, J. Entropy feature based on 2D Gabor wavelets for JPEG steganalysis. In Proceedings of the International Conference on Security, Privacy and Anonymity in Computation, Communication and Storage, Zhangjiajie, China, 16-18 November 2016; pp. 59-72.

11. Karimi, H.; Shayesteh, M.G.; Akhaee, M.A. Steganalysis of JPEG images using enhanced neighbouring joint density features. IET Image Process. 2015, 9, 545-552. [CrossRef]

12. Jyothy, T.S.; Sreelatha, G.; Pradeep, R.; Sajith, V. Texture-Based Multiresolution Steganalytic Features for Spatial Image Steganography. In Proceedings of the 2019 International Conference on Smart Systems and Inventive Technology (ICSSIT), Tirunelveli, India, 27-29 November 2019; pp. 966-971.

13. Liu, Q.; Sung, A.H.; Xu, J.; Ribeiro, B.M. Image complexity and feature extraction for steganalysis of LSB matching steganography. In Proceedings of the 18th International Conference on Pattern Recognition (ICPR'06), Hong Kong, China, 20-24 August 2006; Volume 2, pp. 267-270.

14. Lin, Q.; Liu, J.; Guo, Z. Local ternary pattern based on path integral for steganalysis. In Proceedings of the 2016 IEEE International Conference on Image Processing (ICIP), Phoenix, AZ, USA, 25-28 September 2016; pp. 2737-2741.

15. Shi, Y.Q.; Chen, C.; Xuan, G.; Su, W. Steganalysis versus splicing detection. In International Workshop on Digital Watermarking; Springer: Berlin/Heidelberg, Germany, 2007; pp. 158-172.

16. Ojala, T.; Pietikäinen, M.; Harwood, D. A comparative study of texture measures with classification based on featured distributions. Pattern Recognit. 1996, 29, 51-59. [CrossRef]

17. Costa, A.F.; Humpire-Mamani, G.; Traina, A.J.M. An efficient algorithm for fractal analysis of textures. In Proceedings of the 2012 25th SIBGRAPI Conference on Graphics, Patterns and Images, Ouro Preto, Brazil, 22-25 August 2012; pp. 39-46.

18. Haralick, R.M.; Shanmugam, K.; Dinstein, I.H. Textural features for image classification. IEEE Trans. Syst. Man. Cybern. 1973, SMC-3, 610-621. [CrossRef]

19. Clausi, D.A. An analysis of co-occurrence texture statistics as a function of grey level quantization. Can. J. Remote Sens. 2002, 28, 45-62. [CrossRef]

20. Haralick, R.M. Statistical and structural approaches to texture. Proc. IEEE 1979, 67, 786-804. [CrossRef]

21. Mohanaiah, P.; Sathyanarayana, P.; GuruKumar, L. Image texture feature extraction using GLCM approach. Int. J. Sci. Res. Publ. 2013, 3, 1-5.

22. Sharifi, K.; Leon-Garcia, A. Estimation of shape parameter for generalized Gaussian distributions in subband decompositions of video. IEEE Trans. Circuits Syst. Video Technol. 1995, 5, 52-56. [CrossRef]

23. Friedman, J.; Hastie, T.; Tibshirani, R. The Elements of Statistical Learning; Springer Series in Statistics New York; Springer: New York, NY, USA, 2001; Volume 1.

24. Lowd, D.; Domingos, P. Naive Bayes models for probability estimation. In Proceedings of the 22nd International Conference on Machine Learning, Bonn, Germany, 7-11 August 2005; pp. 529-536.

25. Yang, Z.; Wang, K.; Ma, S.; Huang, Y.; Kang, X.; Zhao, X. Istego100k: Large-scale image steganalysis dataset. arXiv 2019, arXiv:1911.05542.

26. Qin, J.; Xiang, X.; Deng, Y.; Li, Y.; Pan, L. Steganalysis of highly undetectable steganography using convolution filtering. Inf. Technol. J. 2014, 13, 2588. [CrossRef] 